\title{
Identification of Function LncRNAs Associated With Hepatocellular Carcinoma
}

\author{
Xiaolei Wang \\ Qingdao Third People's Hospital \\ Jun Fang \\ Qingdao Third People's Hospital \\ An Wang \\ Qingdao Third People's Hospital \\ Wuhui Zhu ( $\nabla$ zhuwuhui@126.com) \\ Qingdao Third People's Hospital
}

\section{Research Article}

Keywords: hepatocellular carcinoma, survival analysis, prognosis

Posted Date: March 8th, 2022

DOI: https://doi.org/10.21203/rs.3.rs-1303494/v2

License: (c) (i) This work is licensed under a Creative Commons Attribution 4.0 International License. Read Full License 


\section{Abstract \\ Background}

Hepatocellular carcinoma (HCC) is one of the most common malignant tumors of the digestive tract. New diagnostic and prognostic biomarkers are urgently needed for HCC. To investigate potential pathogenic and prognostic long noncoding RNAs (IncRNAs) associated with HCC, we conduct this study.

\section{Material and Methods}

Gene expression matrix were obtained from TCGA and GEO databases. The overlapping differentially expressed genes (DEGs) between TCGA and GEO databases were analyzed using the limma package. Then we constructed mRNA-IncRNA-miRNA network. In the end, Gene ontology (GO), Kyoto Encyclopedia of Genes and Genomes (KEGG) pathway analysis and survival analysis were performed to find hub IncRNAs related to diagnosis and prognosis of HCC.

\section{Results}

In total, 299 DEGs including 10 IncRNAs and 289 mRNAs were acquired. The mRNA-IncRNA-miRNA network comprised of four IncRNAs (GSEC, LINC00844, LINC01146 and MIR99AHG), 49 miRNAs and 25 mRNAs. GO and KEGG analysis shown that four IncRNAs were mainly enriched in cell cycle. In addition, survival analysis demonstrated that there is a significant correlation between GSEC and overall survival rate. Finally, the expression of LINC00844, LINC01146 and MIR99AHG were down-regulated in HCC tissues, and GSEC was up-regulated.

\section{Conclusion}

In summary, four IncRNAs including GSEC, LINC00844, LINC01146 and MIR99AHG may play an important part in the development of HCC. The function of these IncRNAs may have a great effect to the overall survival rate of HCC.

\section{Background}

Hepatocellular carcinoma (HCC) is the most common histological type of primary liver cancer (90\%) and the fourth leading cause of cancerrelated death in the world [1]. TNM staging of tumors has an important value in treatment and diagnosis of tumors, but its predictive value for clinical prognosis is limited. Despite there has a great progress in pathology, epidemiology, and molecular mechanisms at present, there is still room for improvement in predicting the clinical prognosis of HCC. In terms of therapeutic methods, many new methods such as targeted therapy and immunotherapy have emerged. However, therapeutic treatments only benefit part of patients. Thus, novel molecular markers associated with diagnosis and prognosis are still urgently needed to ameliorate bad outcome of HCC. It's important to discriminate the high-risk patients in the early stage. Developing effective treatment methods is also critical to reduce the high reoccurrence of HCC.

An increasing body of research demonstrated that IncRNAs play a more and more important part in various cancers, including breast cancer [2], gallbladder cancer [3], colorectal carcinoma [4], and HCC [5]. The abnormal expression of IncRNAs in HCC is closely associated with oncogenesis, metastasis and prognosis. Some carcinogenic and anticancer IncRNAs associated with HCC have been found [5-7]. Up-regulated IncRNAs, such as ATB, MCM3AP-AS1, PCNAP1 and $\mathrm{H} 19$ have been proved to be closely related to various clinical features and cell phenotypes [5, 8-10]. For instance, m5C-modified H19, which is a IncRNA, may promote the development of carcinoma by recruiting G3BP1 [10]. Of the down-regulated IncRNAs, uc.134 suppresses HCC progression by inhibiting the CUL4A-mediated ubiquitination of LATS1. It also can restrain HCC development by increasing YAPS127 phosphorylation [11]. While cytosporone-B, which is an agonist for Nur77, could promote WFDC21P, then it can inhibit HCC through WFDC21P [12].

Many studies had demonstrated that IncRNAs had various effects in HCC. Competing endogenous RNA (ceRNA) network is one of the most popular mechanism, that is to say, mRNA-IncRNA-miRNA network. The ceRNA network hypothesis considered that IncRNAs are important endogenous molecular sponges, which can competitively integrate miRNAs. This process can regulate mRNA expression levels. Salmena et al. first proposed this hypothesis [13]. Lots of researches have demonstrated that mRNA-IncRNA-miRNA network plays an important part in tumorigenesis, development and metastasis. For instance, IncRNA SNHG8 acted as a sponge of miR-149 which can balance the tumor inhibiting effects of miR-149 in HCC [14]. In addition, LINC01093/miR-96-5p/ZFAND5/NF-kB signaling axis may have an important role in the pathogenesis of HCC [15]. Thus, the ceRNA network is beneficial to gain insight into intricated gene interactions in HCC.

Although there are many researches had studied the diagnostic capability of IncRNAs, lots of IncRNAs are worth of further exploration [16]. Take the important function of IncRNAs of HCC into consideration, the purpose of this research was to explore the role of IncRNAs in HCC patients.

Page $2 / 13$ 
Gene expression matrix of mRNA and IncRNA were took into integrated analysis. We constructed ceRNA network on the basis of ceRNA theory. In the first place, DEGs were acquired from HCC and normal tissue samples which were obtained from GEO and TCGA database. Then the overlapping DEGs between two databases were obtained by using the limma package. In the end, in order to analyze the underlying roles of IncRNAs, we constructed lots of analysis, such as mRNA-IncRNA-miRNA network, GO analysis, KEGG pathway analysis, protein-protein interaction (PPI) network and survival analysis. This research may provide novel views to the molecular mechanism of HCC.

\section{Material And Methods}

\subsection{Data source}

The microarray data of HCC was obtained from GEO (www.ncbi.nlm.nih.gov/geo) and TCGA database. First, we got the eligible data from GEO database. It was considered eligible when studies meet the following criteria: (1) Studies including HCC and adjacent normal tissues. (2) Studies containing matched platform information which can be used for further analyze. On the basis of these criteria, two independent datasets of HCC were acquired from GEO database. The detailed information of each dataset is shown in Table 1. Then, clinical data of HCC patients and the corresponding gene expression profile data were downloaded from TCGA. Clinical data of these HCC patients is displayed in Table 2. Our present study was performed as shown in the flow chart (Fig. 1).

\subsection{Reannotation of raw data}

In the begining, the raw data and expression matrix were obtained from GEO datasets. The probes which had unique gene were retained for analysis. Then, the retained probes should match the corresponding IncRNAs or mRNAs retrieved from the GENCODE. We used average expression values when lots of probes corresponded to one gene.

\subsection{Identification of differential expression analysis}

The raw data were transformed into expression matrix using Affy R package. Then, the limma package was used to identify DEGs from TCGA and GEO datasets. A $\mid \log \mathrm{FCl}>1$ and adjust $P$ value $<0.05$ was regarded as significantly different. The intersected DEGs among TCGA, GSE101728 and GSE93789 datasets were obtained. And then these DEGs were used to do further analyze.

\subsection{Construction of mRNA-IncRNA-miRNA network}

To analyze whether these IncRNAs and miRNAs can construct ceRNA network, we used DIANA Tools to collect IncRNA-targeted miRNAs. MiRDB was used to predict miRNA-mRNA interactions. The DEGs were used to make mRNA-IncRNA-miRNA network. We used the Cytoscape display mRNA-IncRNA-miRNA network.

\subsection{Functional enrichment analysis}

GO and KEGG pathway enrichment analysis were performed using cluster Profiler package. Protein-protein interaction (PPI) network were conducted using STRING v 9.1 (http://string-db.org/).

\subsection{Survival analysis}

In order to analyze if the hub IncRNAs and corresponding mRNAs were associated to the prognosis of HCC patients, Kaplan-Meier (KM) analysis was constructed using the survival package. All cases were split into two groups using the median value of a gene's expression. It was considered as statistically significant when $P$ value was less than 0.05 .

\subsection{Data availability}

TCGA (The Cancer Genome Atlas) dataset is available using the following link: https://portal.gdc.cancer.gov/. GEO (Gene Expression Omnibus) dataset is available using the following link: https://www.ncbi.nlm.nih.gov/geo/.

\section{Results}

\subsection{Characteristics of gene expression from GEO}

The gene expression data (GSE93789, GSE101728) were obtained from the GEO data repository (http://www.ncbi.nlm.nih.gov/geo/). After reannotation, 33591 genes in GSE93789 (GPL16956) and 46102 genes in GSE101728 (GPL21047) were obtained. On the basis of the criteria of |log $\mathrm{FC} \mid>1$ and $p$-adjusted value<0.05, gene expression data were analyzed for differentially expressed genes using limma package. The GSE93789 had 4553 DEGs, containing 2735 up-regulated and 1818 down-regulated genes. The GSE101728 had 1599 DEGs, containing 657 upregulated and 942 down-regulated genes. The particular information of GSE93789 and GSE101728 data set and the number of DEGs acquired 
from the two data sets are displayed in Table 1. We constructed volcano plots to show the significantly DEGs (Fig. 2). We used the heatmap to display the gene expression changes (Fig. 3).

\subsection{Clinical data of HCC and the intersected DEGs}

The clinical data of HCC patients were obtained from TCGA database. The information of HCC patients mainly containing sex, age, TNM stage, tumor grade, Child-Pugh, residual tumor classification and Ishak fibrosis score were collected, as shown in Table 2. Limma package was used to analyze DEGs on the basis of the criteria of $\mid$ Log FC| $>1$ and adjust $P$ value $<0.05$. In summary, 148 IncRNAs with 68 up-regulated and 80 downregulated, 2181 mRNAs with 728 up-regulated and 1453 down-regulated were screened. Venn diagram demonstrates intersected genes both coming from GEO and TCGA. Totally, 289 differentially expressed mRNAs and 10 differentially expressed IncRNAs were acquired (Fig. 4 and Table 3).

\section{3 ceRNA network}

In order to analyze if these hub IncRNAs and mRNAs existing mRNA-IncRNA-miRNA network, we used the intersected IncRNAs and mRNAs to perform mRNA-IncRNA-miRNA network. Ultimately, four IncRNAs, forty-nine miRNAs and twenty-five mRNAs were involved in mRNA-IncRNAmiRNA network (Fig. 5).

\subsection{GO, KEGG pathway analysis, and PPI network}

In order to analyze the underlying biological roles of the hub genes contained in the mRNA-IncRNA-miRNA network, we performed GO and KEGG analysis (Fig. 6). The outcome shown that the biological functions mainly related to nuclear division, sister chromatid segregation, organelle fission and chromosome segregation, while the molecular functions gathered in monooxygenase activity, oxidoreductase activity, iron ion binding and heme binding. The KEGG pathway analysis demonstrated that most of the genes were related to cell cycle, p53 signaling pathway, oocyte meiosis, complement and coagulation cascades and progesterone-mediated oocyte maturation. Furthermore, differentially expressed mRNAs were used to construct PPI network by STRING. The outcome demonstrated that most genes are associated with cell cycle.

\subsection{Prognostic value of hub IncRNAs and mRNAs in patients with HCC}

In order to find out whether the hub IncRNAs and mRNAs involved in the mRNA-IncRNA-miRNA network were related to the prognosis of HCC patients, we analyzed the hub gene expression and the survival data in TCGA. Because some patients didn't have intact information to do survival analysis, 415 cases were obtained in the end. In the four hub IncRNAs, the result demonstrated that IncRNA GSEC was obviously significant associated with the overall survival (OS) $(P<0.05)$. The expression of GSEC was negatively correlated with survival time of $\mathrm{HCC}$. In the 25 hub mRNAs, the result shown that 12 mRNAs were related to the OS of HCC: $\mathrm{B} 3 \mathrm{GNT} 5(P<0.001), \operatorname{SORBS} 2(P=0.037), \mathrm{ENAH}(P=0.003), \mathrm{SUCO}(P=0.004)$, $\operatorname{ESR} 1(P=0.006), \operatorname{FAT} 4(P=0.047), \operatorname{SLC} 38 A 2(P=0.012), \operatorname{MASP} 1(P=0.031), \operatorname{GHR}(P=0.007), \operatorname{PARPBP}(P=0.002), \operatorname{RRM} 2(P<0.001)$ and

MMS22L $(P<0.001)$. The high levels of SORBS2, ESR1, FAT4, MASP1 and GHR predicted good prognosis, but seven mRNAs had a negative correlation with the OS of HCC. The results of KM analysis are displayed in Figure 7.

\section{Discussion}

LncRNAs are becoming more and more popular with the rapid development of high-throughput sequencing and bioinformatic technologies. At present, we obtained the gene expression of HCC from GEO and TCGA. Then we explored the hub IncRNAs of HCC. We constructed mRNAIncRNA-miRNA network, GO and KEGG pathway analysis, PPI network and survival analysis. In the end, the result shown that four IncRNAs including GSEC, LINC00844, LINC01146 and MIR99AHG may have a relation with HCC development. The underlying roles of these hub IncRNAs might be related to the $\mathrm{OS}$ of $\mathrm{HCC}$.

There are many integrated bioinformatics which can be used to find DEGs in HCC. With the rapid development of bioinformation technology, lots of novel genes have been found. In order to find novel IncRNAs and mRNAs associated with the prognosis of HCC, the RNA expression data from GEO was analyzed. In addition, different individual had different gene expression level of the same gene. In order to minimize the error of different individual, we only analyzed paired tissue samples in GEO. After we obtained the DEGs from TCGA, intersected DEGs from GEO and TCGA were used to further analyze. In summary, 10 co-differentially expressed IncRNAs and 289 mRNAs were used to continue next investigation.

More and more studies have shown that ceRNAs have great effects in HCC $[17,18]$. To explore the molecular mechanisms of these hub IncRNAs, we constructed mRNA-IncRNA-miRNA network. In the end, the mRNA-IncRNA-miRNA network included 4 IncRNAs (GSEC, LINC00844, LINC01146 and MIR99AHG), 49 miRNAs and 25 mRNAs. We found that the hub IncRNAs can affect mRNAs expression through competitively uniting miRNA.

MIR99AHG may suppress lung adenocarcinoma's proliferation and metastasis and it can promote autophagy [19]. Studies showing that not only MIR99AHG but also miR-99a expression level was positively related to the survival of lung adenocarcinoma patients. Furthermore, when the expression of MIR99AHG of H1299 cells was overexpressed, the nuclear expression of ANXA2 was obviously decreased. The expression of 
MIR99AHG was obviously up-regulated in gastric cancer [20]. In addition, MIR99AHG was obviously overexpressed in pancreatic cancer. It can promote the proliferation and metastasis of pancreatic cancer. Studies demonstrated that up-regulated MIR99AHG was induced by transcription factor FOXA1 [21].

LINC00844 was found to be significantly decreased in HCC tissue and was correlated with poor tumor characteristics, such as portal vein invasion, high a-fetoprotein (AFP), and a high rate of tumor recurrence [22]. Studies demonstrated that LINC00844 had an important role in suppressing tumor development and metastasis [23, 24]. Besides, LINC00844 is important in facilitating AR binding to the chromatin. In the end, LINC00844 works partly by activating the expression level of NDRG1 which is an important tumor metastasis suppressor. It has been suggested that LINC01146 was a hub IncRNA in both subtypes of chronic rhinosinusitis with nasal polyps [25]. However, there was no researches have demonstrated the role of LINC01146 in HCC up to now.

Unlike LINC00844 and MIR99AHG ,the expression level of GSEC can be up-regulated in many tumors, including colon cancer [26], breast cancer [27], lung cancer [28] and osteosarcoma [29]. Studies have revealed that GSEC has an important influence in cell migration of colon cancer by suppressing the role of DHX36 through its G-quadruplex structure [26]. Moreover, GSEC was significantly up-regulated in triple negative breast cancer [27]. GSEC functioned as a underlying oncogene and it can promote AXL-mediated TNBC progression by sponging miR-202-5p. In addition, High expression level of GSEC promoted the proliferating and migratory capacity, and suppressed the apoptosis of osteosarcoma [29].

There are some limitations in the present study. First of all, this study only contains two GEO data sets, which are both noncoding RNA data. Then, although we acquire the expression matrix of genes by using probe reannotation method, some genes which can't match the probes will not be found because of the platform focused on noncoding RNA data. But the aim of this research was to find hub IncRNA of HCC patients, so the roles of 4 IncRNAs could not be further explored because the mRNAs which were used to analyze were acquired from noncoding RNA data. That is to say, the hub mRNAs were associated with IncRNAs. In total, the results of our analysis are precise and reliable, and it can provide more valuable information to the clinical work. Although we find 4 IncRNAs were statistically related to OS of HCC, more experiments are needed to validate the function of these hub IncRNAs.

\section{Conclusion}

In total, four IncRNAs including GSEC, LINC00844, LINC01146 and MIR99AHG may play an important part in the development of HCC. The function of these IncRNAs may have a great effect to the overall survival rate of HCC.

\section{Declarations}

Author contributions WZ: conception and design. XW: development of methodology, analysis and writing of the manuscript. JF and AW: review of the manuscript. No funding was received.

Funding No funding was received.

Conflict of interest The authors declare that there is no conflict of interests.

Ethical approval Not applicable.

Consent to publish All authors have given their consent to participate in this report and submit it to Molecular Biology Reports.

\section{References}

1. Hepatocellular carcinoma. Nature reviews Disease primers. $2021 ; 7(1): 7$.

2. Liang Y, Song X, Li Y, et al. LncRNA BCRT1 promotes breast cancer progression by targeting miR-1303/PTBP3 axis. Mol Cancer. 2020; 19(1):85.

3. Hu YP, Jin YP, Wu XS, et al. LncRNA-HGBC stabilized by HuR promotes gallbladder cancer progression by regulating miR-502-3p/SET/AKT axis. Mol Cancer. 2019; 18(1):167.

4. Wang FW, Cao $\mathrm{CH}$, Han $\mathrm{K}$, et al. APC-activated long noncoding RNA inhibits colorectal carcinoma pathogenesis through reduction of exosome production. J Clin Invest. 2019; 129(2):727-43.

5. Yuan JH, Yang F, Wang F, et al. A long noncoding RNA activated by TGF- $\beta$ promotes the invasion-metastasis cascade in hepatocellular carcinoma. Cancer Cell. 2014; 25(5):666-81.

6. Song X, Du R, Gui H, et al. Identification of potential hub genes related to the progression and prognosis of hepatocellular carcinoma through integrated bioinformatics analysis. Oncol Rep. 2020; 43(1):133-46. 
7. Yu B, Ding Y, Liao X, Wang C, Wang B, Chen X. Overexpression of PARPBP Correlates with Tumor Progression and Poor Prognosis in Hepatocellular Carcinoma. Dig Dis Sci. 2019; 64(10):2878-92.

8. Wang Y, Yang L, Chen T, et al. A novel IncRNA MCM3AP-AS1 promotes the growth of hepatocellular carcinoma by targeting miR-1945p/FOXA1 axis. Mol Cancer. 2019; 18(1):28.

9. Feng J, Yang G, Liu Y, et al. LncRNA PCNAP1 modulates hepatitis B virus replication and enhances tumor growth of liver cancer. Theranostics. 2019; 9(18):5227-45.

10. Sun Z, Xue S, Zhang M, et al. Aberrant NSUN2-mediated mC modification of H19 IncRNA is associated with poor differentiation of hepatocellular carcinoma. Oncogene. 2020; 39(45):6906-19.

11. Ni W, Zhang Y, Zhan Z, et al. A novel IncRNA uc.134 represses hepatocellular carcinoma progression by inhibiting CUL4A-mediated ubiquitination of LATS1. J Hematol Oncol. 2017; 10(1):91.

12. Guan Y-F, Huang Q-L, Ai Y-L, et al. Nur77-activated IncRNA WFDC21P attenuates hepatocarcinogenesis via modulating glycolysis. Oncogene. 2020; 39(11):2408-23.

13. Salmena L, Poliseno L, Tay Y, Kats L, Pandolfi PP. A ceRNA hypothesis: the Rosetta Stone of a hidden RNA language? Cell. 2011; 146(3):35358.

14. Dong J, Teng F, Guo W, Yang J, Ding G, Fu Z. IncRNA SNHG8 Promotes the Tumorigenesis and Metastasis by Sponging miR-149-5p and Predicts Tumor Recurrence in Hepatocellular Carcinoma. Cellular physiology and biochemistry : international journal of experimental cellular physiology, biochemistry, and pharmacology. 2018; 51(5):2262-74.

15. Zheng Y, Yu K, Huang C, et al. Integrated bioinformatics analysis reveals role of the LINC01093/miR-96-5p/ZFAND5/NF-KB signaling axis in hepatocellular carcinoma. Exp Ther Med. 2019; 18(5):3853-60.

16. Lim LJ, Wong SYS, Huang F, et al. Roles and Regulation of Long Noncoding RNAs in Hepatocellular Carcinoma. Cancer Res. 2019; 79(20):5131-39.

17. Wang H, Huo X, Yang X-R, et al. STAT3-mediated upregulation of IncRNA HOXD-AS1 as a ceRNA facilitates liver cancer metastasis by regulating SOX4. Mol Cancer. 2017; 16(1):136.

18. Li X, Ding J, Wang X, Cheng Z, Zhu Q. NUDT21 regulates circRNA cyclization and ceRNA crosstalk in hepatocellular carcinoma. Oncogene. 2020; 39(4):891-904.

19. Han C, Li H, Ma Z, et al. MIR99AHG is a noncoding tumor suppressor gene in lung adenocarcinoma. Cell Death Dis. $2021 ; 12(5): 424$.

20. Meng Q, Wang X, Xue T, Zhao Q, Wang W, Zhao K. Long noncoding RNA MIR99AHG promotes gastric cancer progression by inducing EMT and inhibiting apoptosis via miR577/FOXP1 axis. Cancer Cell Int. 2020; 20:414.

21. Xu J, Xu W, Yang X, Liu Z, Zhao Y, Sun Q. LncRNA MIR99AHG mediated by FOXA1 modulates NOTCH2/Notch signaling pathway to accelerate pancreatic cancer through sponging miR-3129-5p and recruiting ELAVL1. Cancer Cell Int. 2021; 21(1):674.

22. Lin B, He H, Zhang Q, et al. Long non-coding RNA00844 inhibits MAPK signaling to suppress the progression of hepatocellular carcinoma by targeting AZGP1. Annals of translational medicine. 2020; 8(21):1365.

23. Lingadahalli S, Jadhao S, Sung YY, et al. Novel IncRNA Regulates Prostate Cancer Cell Migration and Invasion through AR Signaling. Molecular cancer research : MCR. 2018; 16(12):1865-78.

24. Qiu K, Zheng Z, Huang Y. Long intergenic noncoding RNA 00844 promotes apoptosis and represses proliferation of prostate cancer cells through upregulating GSTP1 by recruiting EBF1. J Cell Physiol. 2020; 235(11):8472-85.

25. Wang M, Bu X, Luan G, et al. Distinct type 2-high inflammation associated molecular signatures of chronic rhinosinusitis with nasal polyps with comorbid asthma. Clinical and translational allergy. 2020; 10:26.

26. Matsumura K, Kawasaki Y, Miyamoto M, et al. The novel G-quadruplex-containing long non-coding RNA GSEC antagonizes DHX36 and modulates colon cancer cell migration. Oncogene. 2017; 36(9):1191-99.

27. Zhang J, Du C, Zhang L, Wang Y, Zhang Y, Li J. IncRNA GSEC Promotes the Progression of Triple Negative Breast Cancer (TNBC) by Targeting the miR-202-5p/AXL Axis. Onco Targets Ther. 2021; 14:2747-59.

28. Tian Y, Yu M, Sun L, et al. Distinct Patterns of mRNA and IncRNA Expression Differences Between Lung Squamous Cell Carcinoma and Adenocarcinoma. J Comput Biol. 2020; 27(7):1067-78.

29. Liu R, Ju C, Zhang F, et al. LncRNA GSEC promotes the proliferation, migration and invasion by sponging miR-588/ EIF5A2 axis in osteosarcoma. Biochem Biophys Res Commun. 2020; 532(2):300-07.

\section{Tables}

Table 1. Details information of HCC researches and related microarray data sets from GEO 


\begin{tabular}{|c|c|c|c|c|c|c|c|}
\hline GSE & $\begin{array}{l}\text { Total } \\
\text { differentially } \\
\text { expressed } \\
\text { genes }\end{array}$ & $\begin{array}{l}\text { Up- } \\
\text { regulated }\end{array}$ & $\begin{array}{l}\text { Down- } \\
\text { regulated }\end{array}$ & Platform & $\begin{array}{l}\text { Sample } \\
\text { size }\end{array}$ & Age & Sex \\
\hline GSE93789 & 4553 & 2735 & 1818 & GPL16956 & $\begin{array}{l}\text { total:10; } \\
\text { tumor:5; } \\
\text { adjecent:5 }\end{array}$ & $44,45,49,48,40,44,45,49,48,40$ & $\begin{array}{l}\text { Male:8; } \\
\text { Female:2 }\end{array}$ \\
\hline GSE101728 & 1599 & 657 & 942 & GPL21047 & $\begin{array}{l}\text { total:14; } \\
\text { tumor:7; } \\
\text { adjecent:7 }\end{array}$ & $57,57,67,67,58,58,52,52,62,62,54,54,27,27$ & $\begin{array}{l}\text { Male:10; } \\
\text { Female:4 }\end{array}$ \\
\hline
\end{tabular}

Table 2. The clinical data of HCC in TCGA 


\begin{tabular}{|c|c|c|}
\hline Characteristics & Number of cases & Percentages \\
\hline \multicolumn{3}{|l|}{ Sex } \\
\hline Female & 143 & 33.97 \\
\hline Male & 278 & 66.03 \\
\hline \multicolumn{3}{|l|}{ Age } \\
\hline$\otimes 60$ & 226 & 53.68 \\
\hline$\leq 60$ & 195 & 46.32 \\
\hline \multicolumn{3}{|l|}{ Stage } \\
\hline Stage I & 189 & 44.89 \\
\hline Stage II & 97 & 23.04 \\
\hline Stage III & 97 & 23.04 \\
\hline Stage IV & 6 & 1.43 \\
\hline \multicolumn{3}{|l|}{ Histologic grade } \\
\hline $\mathrm{G} 1$ & 60 & 14.25 \\
\hline $\mathrm{G} 2$ & 204 & 48.46 \\
\hline G3 & 137 & 32.54 \\
\hline $\mathrm{G} 4$ & 12 & 2.85 \\
\hline \multicolumn{3}{|l|}{ Topography } \\
\hline $\mathrm{T} 1$ & 201 & 47.74 \\
\hline $\mathrm{T} 2$ & 108 & 25.65 \\
\hline T3 & 93 & 22.09 \\
\hline T4 & 16 & 3.80 \\
\hline \multicolumn{3}{|c|}{ Child-Pugh stage } \\
\hline$A$ & 240 & 57.01 \\
\hline B & 30 & 7.13 \\
\hline C & 1 & 0.24 \\
\hline \multicolumn{3}{|l|}{ Residual tumor } \\
\hline $\mathrm{RO}$ & 364 & 86.46 \\
\hline $\mathrm{R} 1$ & 21 & 4.99 \\
\hline $\mathrm{R} 2$ & 2 & 0.48 \\
\hline $\mathrm{Rx}$ & 25 & 5.94 \\
\hline \multicolumn{3}{|c|}{ Ishak fibrosis score } \\
\hline 0 & 96 & 22.80 \\
\hline 1,2 & 35 & 8.31 \\
\hline 3,4 & 31 & 7.36 \\
\hline 5 & 10 & 2.38 \\
\hline 6 & 75 & 17.81 \\
\hline
\end{tabular}

Table 3. The intersected hub IncRNAs and mRNAs 


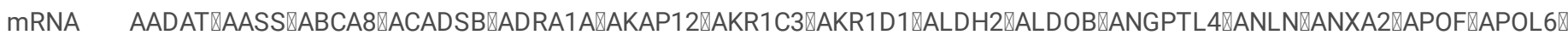

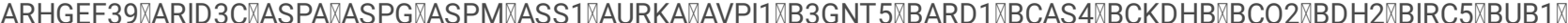

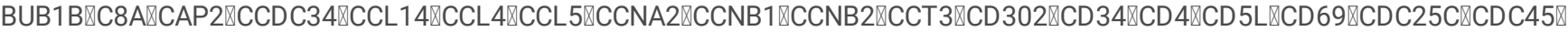

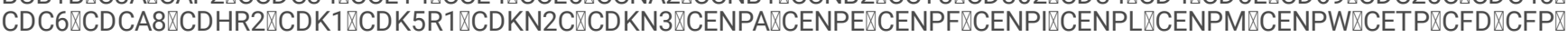

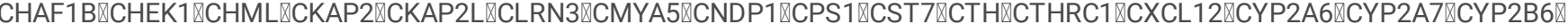

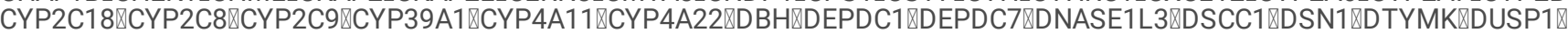

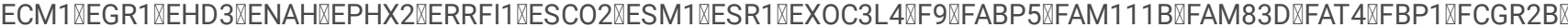

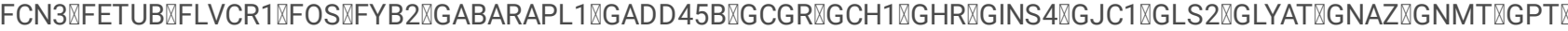

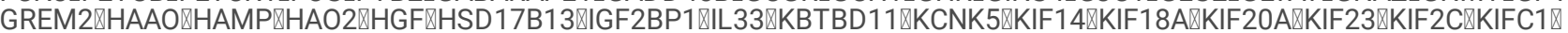

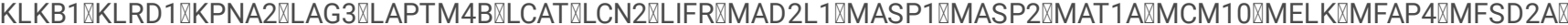

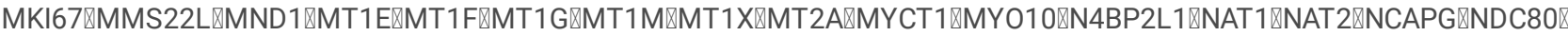

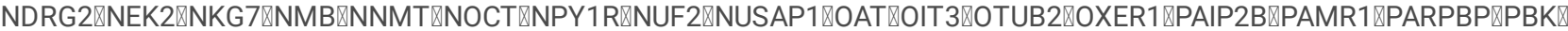

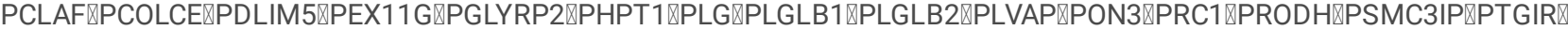

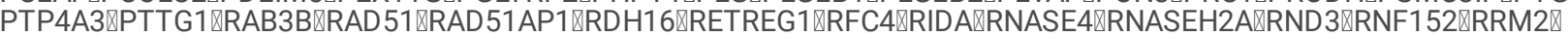

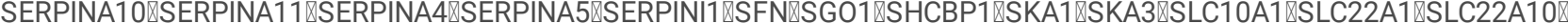

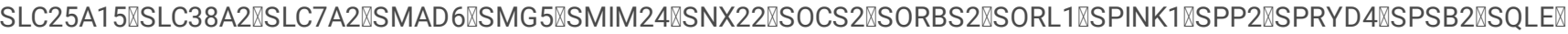

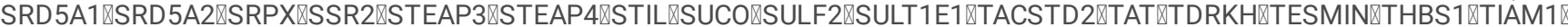

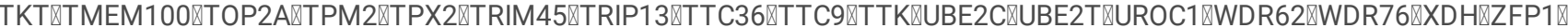
ZG16囚ZKSCAN3囚ZNF607囚ZWINT

\section{Figures}

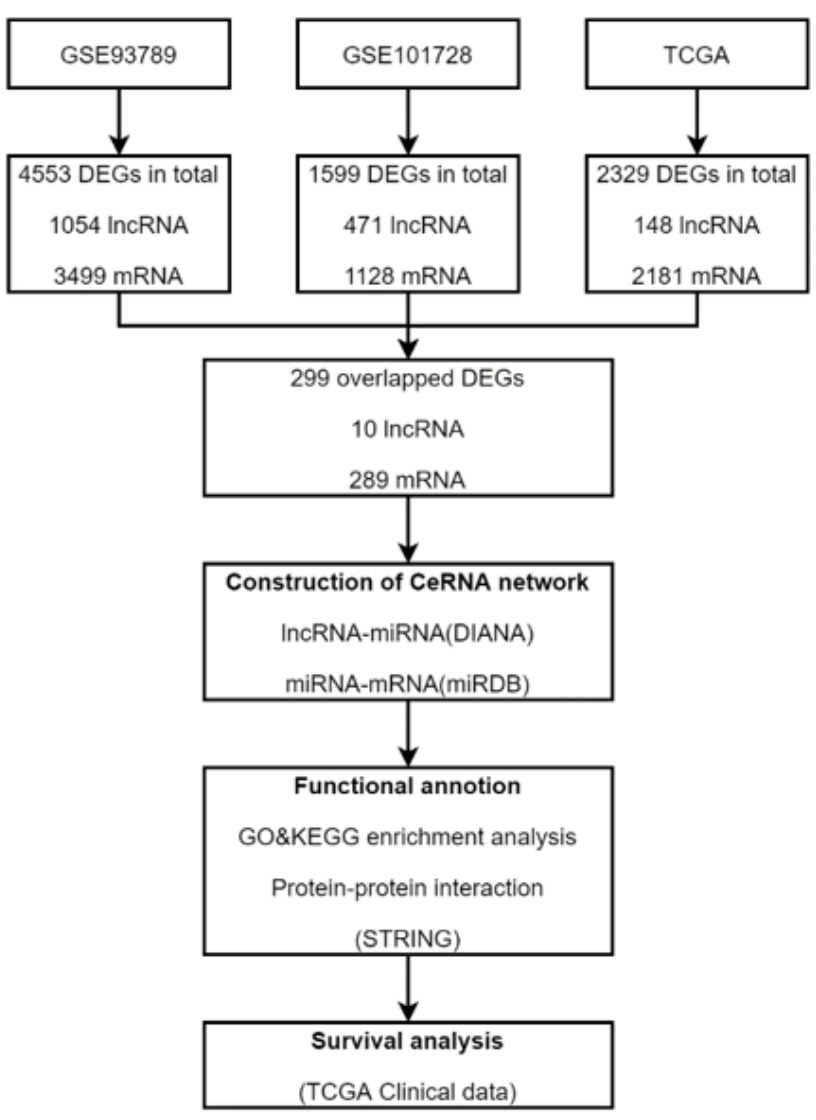

Figure 1

The flow chart shows the process of how to analyze the gene expression data from GEO and TCGA databases. IncRNA, long noncoding RNA; miRDB, microRNA database; miRNA, microRNA; mRNA, messenger RNA; TCGA, The Cancer Genome Atlas 

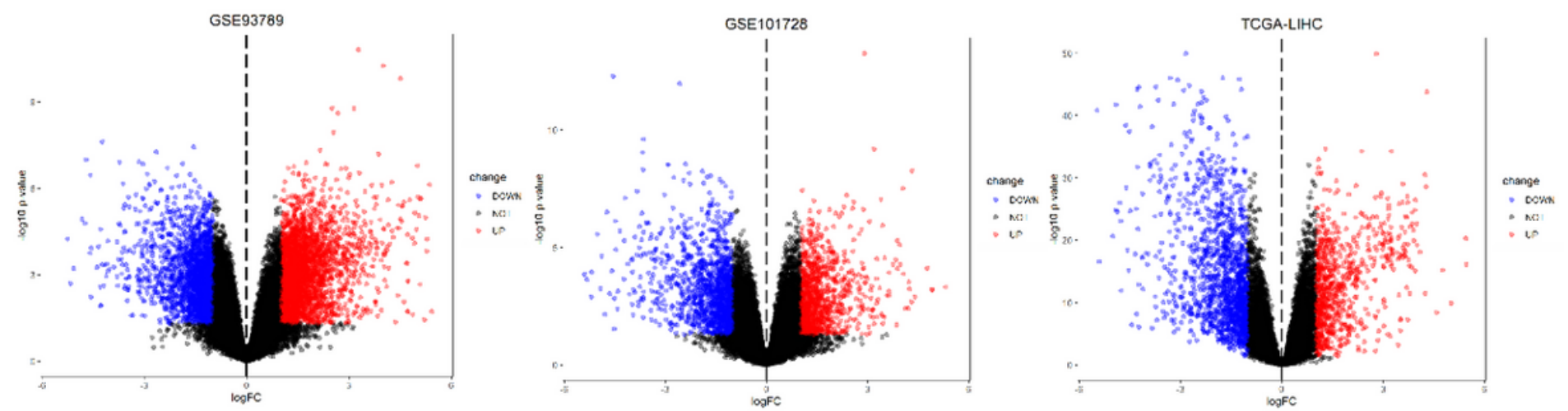

\section{Figure 2}

Volcano plots of DEGs in GSE93789, GSE10728 and TCGA database. The red dots and the blue dots represent the up-regulated and downregulated genes respectively

(A)

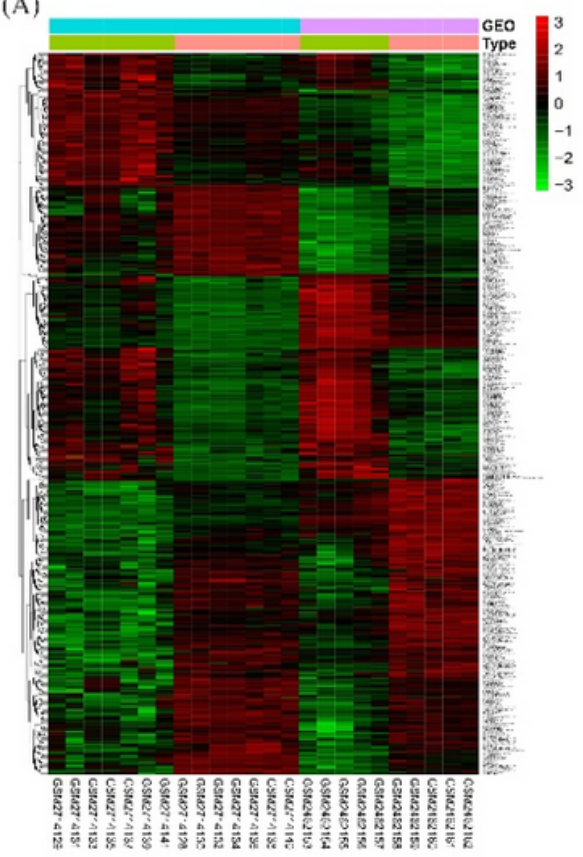

(B)

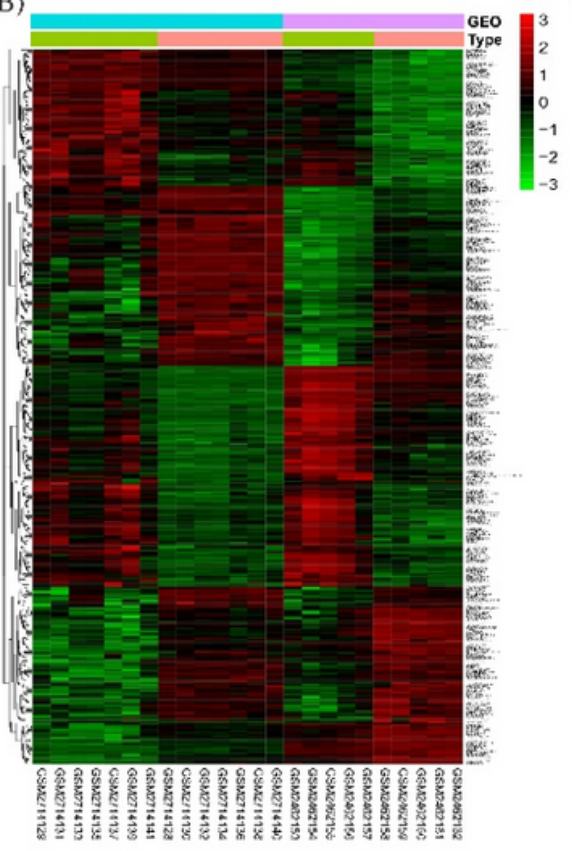

(C)

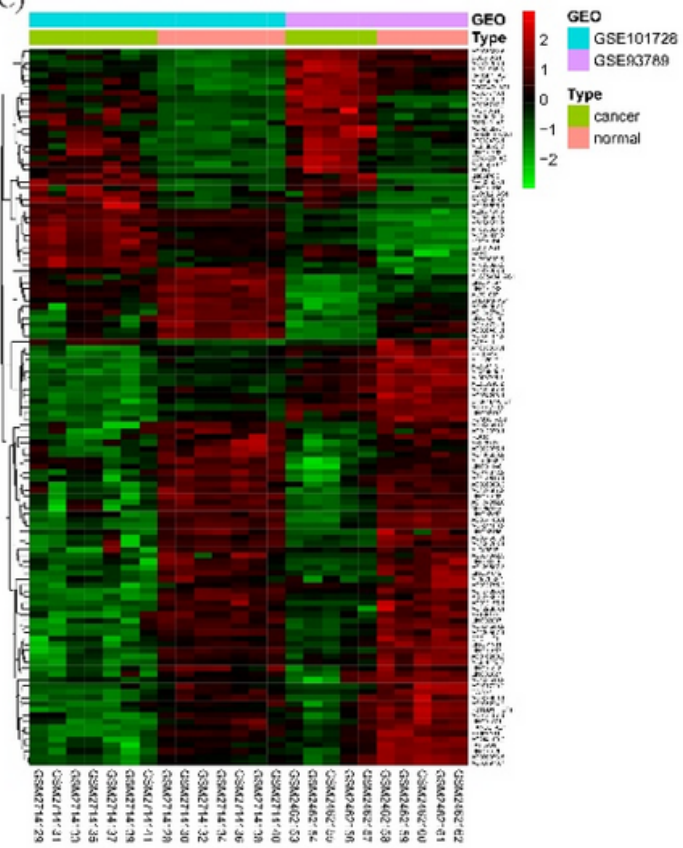

Figure 3

Heatmap of DEGs in GSE93789 and GSE101728 data set. A, total DEGs. B, IncRNAs. C, mRNAs. The red color and the green color represent the up-regulated and down-regulated genes respectively 
(A)

GSE93789

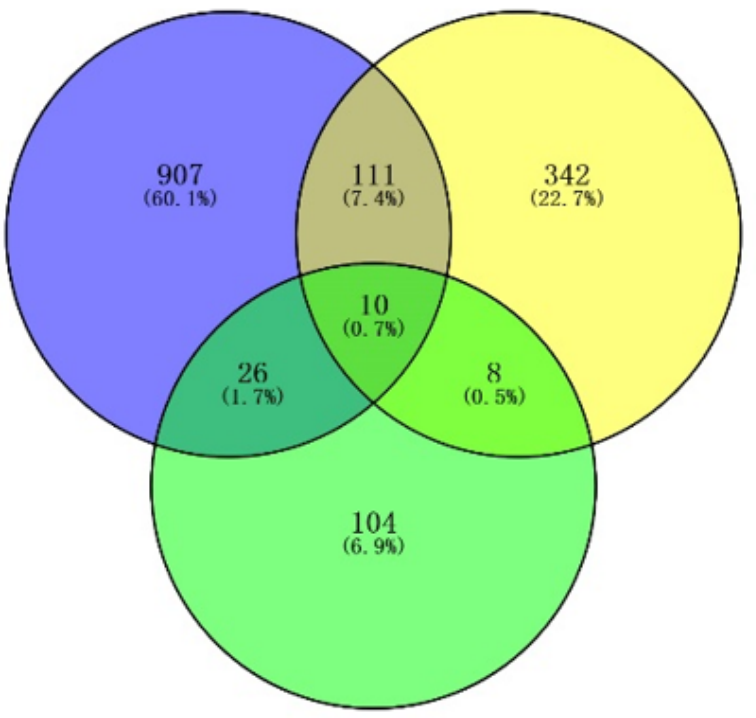

TCGA
(B)

GS1:93789

GSI:101728

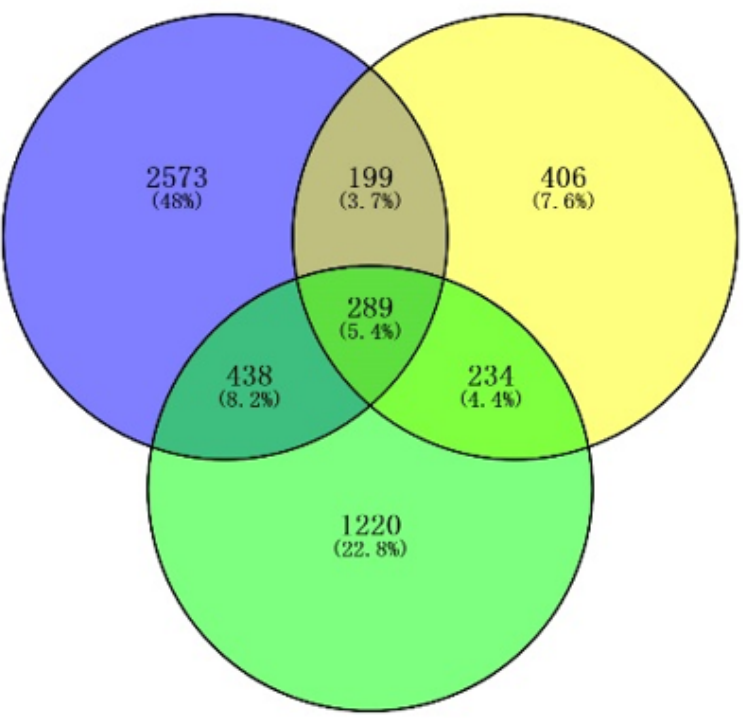

TCGA

\section{Figure 4}

Venny diagram shows the intersected genes both in GEO and TCGA. A, intersected IncRNAs; B, intersected mRNAs

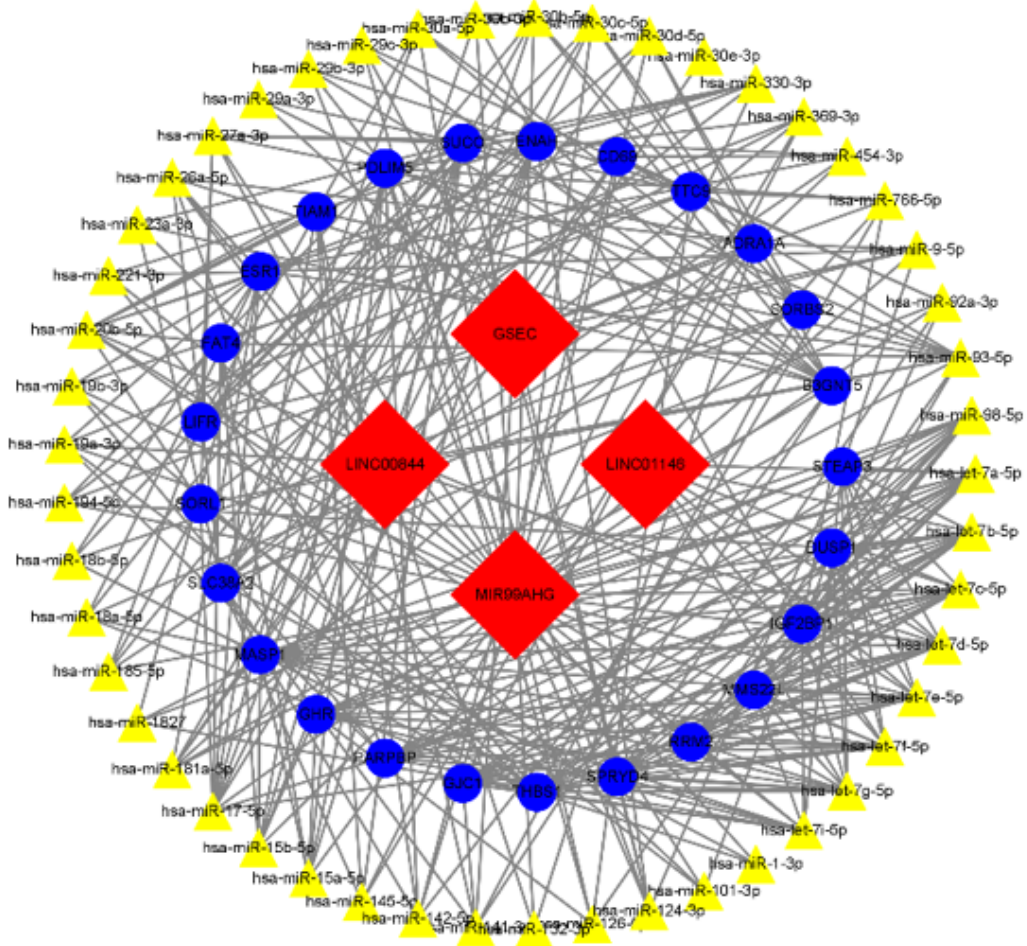

Figure 5

mRNA-IncRNA-miRNA network. Red color means IncRNAs; yellow color means miRNAs; blue color means mRNAs 

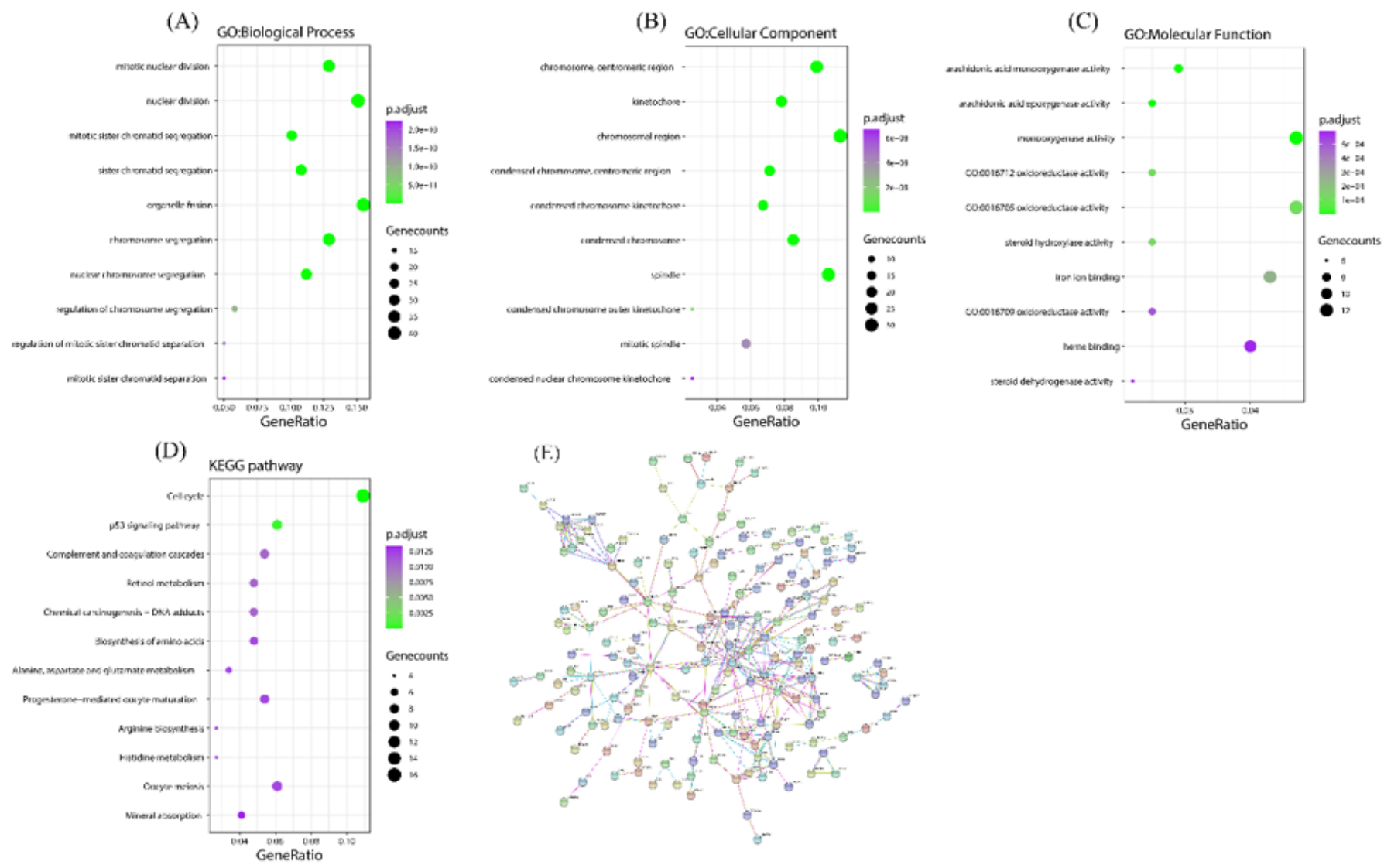

\section{Figure 6}

Functional analysis of DEGs A: GO-biological processes, B: GO-cellular component, C: GO-molecular functions. D, KEGG pathway analysis. E, PPI network 

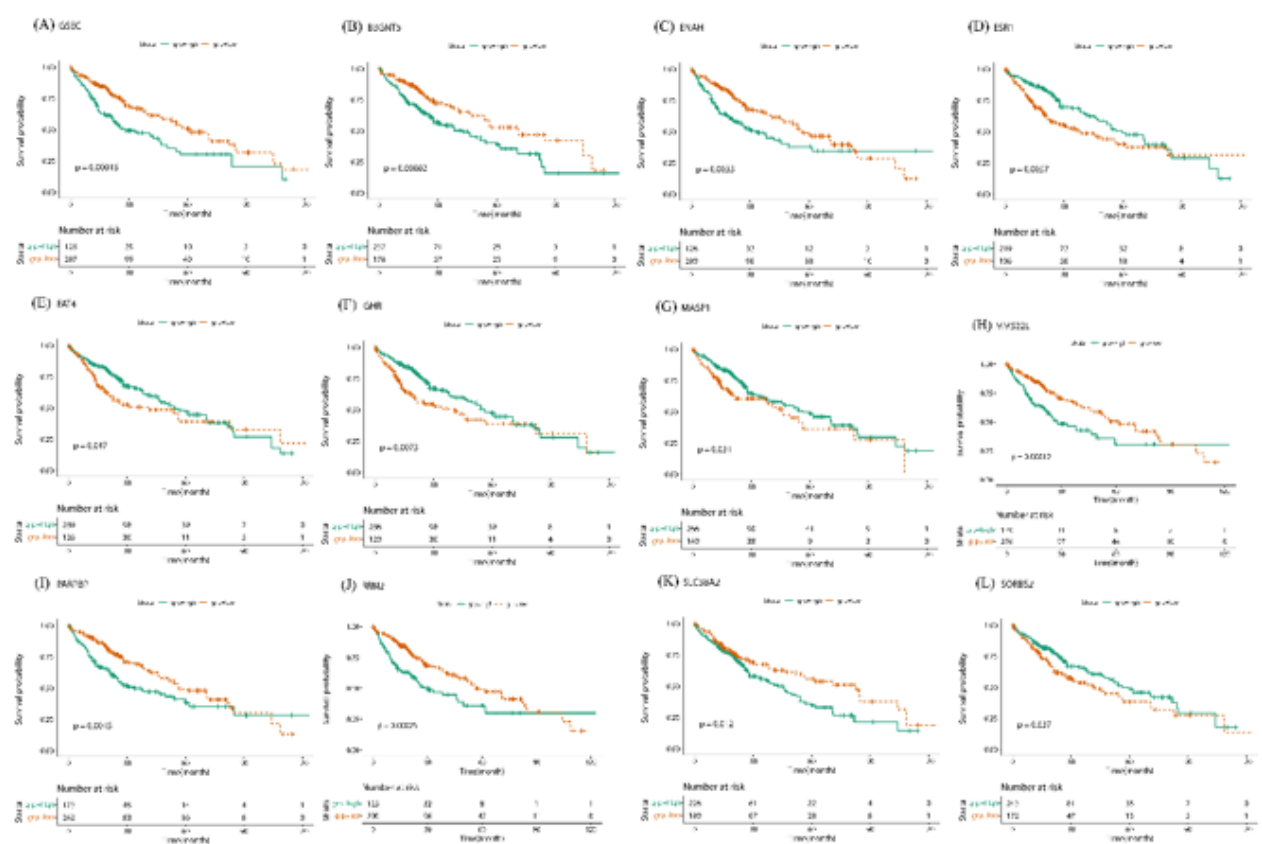

(M) $2 \times 0$
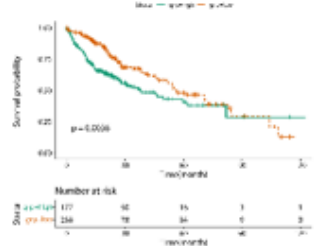

Figure 7

The results of KM analysis of hub genes. We found 13 hub genes were related to the OS of HCC. A, GSEC B, B3GNT5 C, ENAH D, ESR1 E, FAT4 F, GHR G, MASP1 H, MMS22L I, PARPBP J, RRM2 K, SLC38A2 L, SORBS2 M, SUCO 